\section{Herramientas de geolocalización como estrategia didáctica y relacional: una experiencia con el arte latinoamericano en tiempos de pandemia}

\section{Renata Ribeiro dos Santos}

Universidad de Oviedo

\section{Resumen}

El presente artículo describe una propuesta de innovación docente aplicada en la asignatura de Historia del Arte Iberoamericano del Grado de Historia del Arte de la Universidad de Oviedo, durante el periodo de confinamiento. El proyecto se basó en la utilización de la tecnología de geolocalización presente en los dispositivos móviles, para la confección de mapas personales con la identificación de ubicaciones que relacionasen el espacio geográfico de América Latina, los contenidos impartidos en la asignatura y las vivencias y particularidades de cada estudiante. La actividad, que fue concebida para realizarse de manera remota potenciada por el m-learning, se transformó en una práctica indispensable debido a la forzosa y urgente necesidad de adaptar toda la docencia al modo no presencial. Finalmente, además de potenciar los objetivos iniciales de construcción y asimilación de aprendizaje, el proyecto logró establecer lazos de presencialidad entre el estudiantado, en un panorama de fractura de los contactos reales.

Palabras clave: Historia del arte; Cultura latinoamericana; Herramientas de geolocalización; Innovación educativa.
Geolocation tools as a didactic and relational strategy: an experience with Latin American art intimes of pandemic

\section{Abstract}

This article describes a proposal for applied teaching innovation in the History of IberoAmerican Art subject of the History of Art degree at the University of Oviedo during the period of confinement. The project was based on the use of geolocalization technology present in mobile devices, for the creation of personal maps with the identification of locations that relate the geographical space of Latin America, the contents taught in the subject and the experiences and particularities of each student. The activity, which was conceived to be carried out remotely and enhanced by m-learning, became an indispensable practice due to the forced and urgent need to adapt all teaching to the non-attendance mode. Finally, in addition to enhancing the initial objectives of construction and assimilation of learning, the project managed to establish ties of presence among students, in a scenario of fracture of real contacts.

Key words: Art history; Latin American culture; Geolocation tools; Educational innovation.

\section{Introducción}

En el segundo semestre del curso 2019/2020 teníamos contemplado la implementación de un proyecto de innovación docente en la asignatura Arte en Iberoamérica. La asignatura tiene carácter obligatorio y se imparte al Tercer Curso de la carrera de Historia del Arte en la Universidad de Oviedo. El proyecto en cuestión, titulado Herramientas de Geolocalización como recurso Didáctico en la Historia del Arte, fue pensando como una estrategia para lograr una mayor relación entre el estudiantado matriculado en la asignatura con la cultura y el arte de América Latina. Utilizaríamos los mapas, la geografía y las herramientas digitales de geolocalización como herramientas para potencializar estas relaciones. En la primera sesión, cuando presentamos el proyecto, jamás podríamos siquiera imaginar las vicisitudes ocasionadas por la pandemia de la Covid-19, que trastocaron todo lo que conocíamos por normalidad y empujaron nuestra proposición a la configuración

\title{
MAGISTER
}

Vol. 32. Núm. I: (2020). Sección extraordinaria 
de una herramienta relacional, no solo con América Latina y su cultura, pero entre los mismos alumnos y alumnas del grupo y el profesorado.

La idea de lo relacional como concepto está muy presente en el discurso artístico desde finales de la década de los 90 , cuando el teórico y comisario francés Nicolás Bourriaud, definió como relacional el arte producido en aquellas últimas décadas del siglo XX (Bourriaud, 2006). Los artistas en aquellos momentos empezaron a buscar estrategias para salir de lo puramente representacional, incorporando los espectadores a las obras y a las acciones, en un intento de conectarse con la sociedad y su cotidianidad. De acuerdo con Bourriaud, el arte es un "un terreno rico en experimentaciones sociales, como un espacio parcialmente preservado de la uniformidad de los comportamientos. Las obras [...] dibujan, cada una, una utopía de proximidad" (2006, p. 8). Casualmente, durante el proyecto de innovación docente que detallaremos en este escrito, hemos logrado que lo relacional traspasara el límite de lo académico y representacional —común en las aulas de Historia del Arte-, encontrado finalmente una utopía de proximidad.

Al principio, el proyecto nació de la constatación de una relativa falta de contacto y conocimiento de alumnos y alumnas acerca de los acontecimientos históricos y artísticos producidos en América Latina. En muchos casos, sumado a la dificultad de comprensión de los hechos históricos y de su disposición en un eje cronológico, encontramos una incomprensión espacial del territorio latinoamericano, de su proceso de división en el periodo colonial y, consecuentemente, de su conformación actual. Este aspecto necesita especial atención, pues el conocimiento de la geografía y de los procesos de conformación geopolítica es fundamental para analizar cómo estos factores influyen en la construcción de los productos culturales y de las identidades (Alejandre, Ortiz e, Izaguirre, 2018, pp. 1-9).

Particularmente, en el caso de América Latina —enmarcada entre dos océanos, con el Caribe y con la imponente Cordillera de los Andes - resulta imprescindible conocer su paisaje geográfico para entender cómo diferentes etnias modificaron este espacio desde los tiempos precolombinos, pasando por su importancia para los conquistadores europeos, hasta alcanzar las actuales Repúblicas. Asimismo, se ha notado la necesidad de relacionar más directamente los estudiantes con el contexto cultural latinoamericano para estimular la comprensión del Otro cultural, deconstruyendo estereotipos y tópicos. Para lograr este acercamiento hemos optado por aprovecharnos de aquellos aspectos, voluntades o experiencias del ámbito personal de los estudiantes, pasibles de impulsar la creación de un vínculo afectivo y relacional con el medio cultural y artístico latinoamericano.

Uniendo estos tres elementos - el espacio geográfico, los productos culturales y lo afectivo/relacional —, buscamos la posibilidad de crear una visualidad en conjunto, utilizando la tecnología basada en los sistemas de geolocalización, pues esta "permite asociar contenidos digitales (imágenes, vídeos, audios, etc.) a una ubicación geográfica física. [...]. Se pueden elaborar mapas, combinar datos sobre otros eventos, objetos o personas, convertirlos en gráficos, etc." (Gros y Forés, 2013 p. 43). Resumidamente, la geolocalización es una tecnología que utiliza datos de un dispositivo móvil (ordenador, radar, teléfono, etc.) para identificar o situar su ubicación en tiempo real. De forma que, habría que añadir que la elección por el uso de las herramientas de geolocalización se debe a la facilidad y familiaridad que existe en la actualidad, tanto relacionadas a su concepto como a las propias aplicaciones y dispositivos, que se deben, principalmente, al uso extendido de la telefonía móvil.

\section{Geolocalización como herramienta relacional: Método y algunos resultados.}

Como indicamos anteriormente, el proyecto fue aplicado durante el curso 2019/2020, en la asignatura de Historia del Arte de Iberoamérica, de carácter obligatorio en el Tercer Curso, impartida en el $6^{\circ}$ semestre del Grado de Historia del Arte. Consiste en una asignatura de 6 créditos ECTS con 15 horas dedicadas a las clases prácticas, 31 a las teóricas, restando 4 horas de Tutorías Grupales. La matrícula total fue de 34 alumnos y alumnas de los cuales, seis se encontraban realizando estancias nacionales o internacionales.

\section{Los mapas afectivos y la presencialidad}

En nuestra primera sesión práctica, orientamos a los alumnos y alumnas sobre el contenido del proyecto, sus objetivos y los primeros pasos que deberíamos dar.

\section{MAGISTER}

Vol. 32. Núm. I: (2020). Sección extraordinaria 
Todas las orientaciones dadas en las sesiones presenciales posteriormente se colgaban en el Campus Virtual de la asignatura. Se hizo hincapié en el carácter procesual del Proyecto y que, como tal, su consecución y posterior calificación y evaluación, se realizarían de manera continua, a lo largo de todo el semestre académico.

El primer paso para la elaboración del proyecto fue la creación individual de un mapa con la herramienta My Maps, una aplicación para la creación de contenido y uso colaborativo, desarrollada por Google. Diferente de la conocida aplicación Google Maps, una plataforma que permite únicamente el consumo de contenido, en My Maps es posible crear mapas y niveles de información, ubicar puntos y poligonales, medir distancias, etc. (Fundación Telefónica, 2012). Para efectuar ese primer paso sin mayores dificultades y teniendo en cuenta la variedad del estudiantado y sus diferentes niveles de interacción y conocimiento de las herramientas digitales, elaboramos un escrito con las instrucciones generales, un paso a paso, que se subió al Campus Virtual.

Posteriormente a la creación de su "Mapa Personal", cada alumna y alumno debería enviar un correo a la profesora de la asignatura conteniendo el enlace de su mapa. Con estos enlaces, creamos en el Campus Virtual un recurso de contenido web, donde se reunieron y listaron todos los mapas, vinculándolos a los nombres de sus autores y autoras. En total, se crearon 23 mapas, lo que indica una participación de más del $80 \%$ de los y las estudiantes que asistieron el semestre. Creemos que este tipo de actividad auxilia en el incremento de una Educación Personalizada, un modelo educativo que indague acerca de las particularidades y necesidades de cada alumno y alumna, las problematice y que busquen, conjuntamente, soluciones o respuestas (Leiva y Moreno, 2015, p. 2).

Para cada tema y subtema de la asignatura, los y las estudiantes deberían seleccionar un producto o aspecto cultural relacionado con los contenidos impartidos y añadir el respectivo marcador en el mapa. Cada ubicación debería contener una descripción que explicase las motivaciones que los empujaron a elegirla. Asimismo, y aprovechando las posibilidades que brindan My Maps y su conexión con las demás herramientas de Google, cada marcación debería ser suficientemente ilustrada con imágenes o videos.

Orientamos que las búsquedas se basaran en sus relaciones afectivas con América Latina, buscando la construcción de puentes de confinidad con el espacio. Provocamos a que los estudiantes activasen relaciones basadas en la existencia de alguna ascendencia latinoamericana - aprovechando los lazos históricos que unen Asturias a la región-, un viaje realizado o soñado, amigos o amigas que vivan allá, gustos personales, películas, libros, música, etc. Se hizo hincapié de que la selección no se basase únicamente en los elementos culturales de la llamada "alta cultura" monumentos, edificios emblemáticos, artes plásticas y visuales- pero que llegase al terreno más cercano de la cultura popular: arquitectura vernácula, alimentación, bailes, música, subculturas, lenguas, grupos activistas, fiestas populares, creencias y religiones, etc.

Bajo nuestro punto de vista, estimular la inclusión de las manifestaciones y saberes locales en régimen de paridad con las aquellas canónicas e institucionalizadas normalmente las únicas incluidas en los libros didácticos-, ayuda en la elaboración por parte del estudiantado de un horizonte plural y diverso, que crea un conocimiento más complejo y con capacidad de cuestionar normas e imposiciones. Estos preceptos los hemos asimilado de la pedagogía basada en la opción decolonial (Espinosa, 2019; Walsh, 2014), ampliamente difundida en América Latina y que apunta hacia una educación capaz de descolonizar el saber.

La creación de las ubicaciones, como se ha citado anteriormente, se hizo de manera procesual, en la medida que íbamos avanzado con los contenidos de la asignatura. La orientación fue que los alumnos y alumnas realizasen la actividad fuera del horario de las clases, aprovechándonos del uso del móvil, lo que les permite acceder a My Maps en cualquier ambiente. El teléfono móvil como herramienta alternativa de aprendizaje, dentro de lo que se denomina m-learning (mobile learning) (Fombona y Vázquez-Cano, 2017, p. 321), toma este aparato tan difundido y utilizado, sobre todo entre la población más joven, y lo incorpora a las prácticas docentes, tratando de "cerrar la brecha entre el aprendizaje que ocurre en la escuela y el aprendizaje que ocurre en el mundo" (Leiva y Moreno, 2015, p. 2).

\section{MAGISTER}

Vol. 32. Núm. I: (2020). Sección extraordinaria 
Periódicamente, se realizó en las sesiones de prácticas de la asignatura un repaso al desarrollo del proyecto: se discutieron las dificultades y logros y, mientras proyectábamos los mapas en el aula, cada estudiante comentaba al menos uno de sus puntos, explicando de qué forma direccionaba y organizaba su "mapa afectivo / relacional". Hay dos factores destacables de evaluar en estas primeras sesiones. En primer lugar, notamos un incremento del interés del estudiantado hacia el proyecto, lo que corrobora que las actividades personalizadas y con mayor grado de interactividad reflejan en una mayor motivación para integrar el proceso de aprendizaje (Leiva y Moreno, 2015, p. 4). Por otro lado, sin que lo orientáramos explícitamente, algunos alumnos y alumnas empezaron a diseñar sus propuestas de manera temática. Siguiendo las instrucciones de la práctica, fueron moldeando sus ubicaciones hacia sus intereses personales, creando una red de puntos homogeneizada temáticamente. Por ejemplo, crearon mapas con ubicaciones que únicamente tenían marcaciones que resaltaban aspectos de la arquitectura o relacionados a la descendencia o lazos familiares, a la música, al deporte o a la historia de las mujeres (Fig. 1).
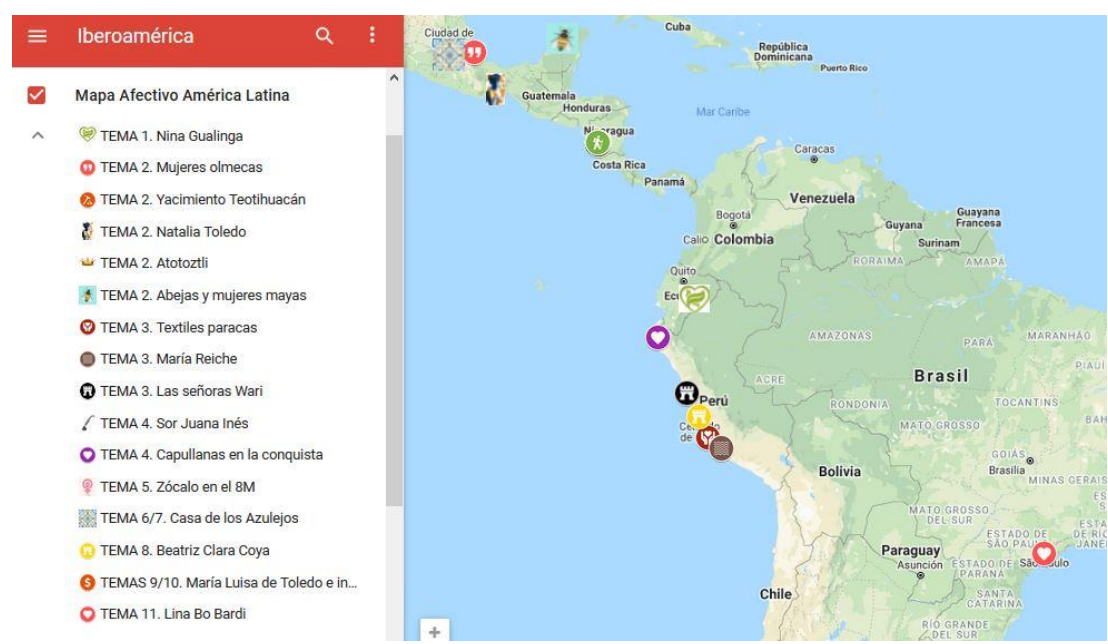

Figura 1. Ejemplo de uno de los Mapas Afectivos/Relacionales temáticos creados en $M y$ Maps.Fuente: Captura de pantalla de My Maps

\section{Los mapas relacionando la no presencialidad}

Con la declaración del Estado de Alarma, a mediados de marzo de 2020, y la suspensión de la actividad presencial hasta finalizar el curso académico, nuestra propuesta pedagógica tuvo que reconfigurarse. Aunque gran parte de la práctica no exigiese la presencialidad y se basaba en m-learning, percibimos que era fundamental mantener la motivación del estudiantado y lograr vincular los contenidos teóricos de la asignatura, que a partir de aquel momento se impartirían globalmente de manera no presencial.

De forma que, previa consulta y aprobación por parte de alumnos y alumnas, decidimos que todas las sesiones de prácticas restantes las dedicaríamos a los Mapas Afectivos/Relacionales. En estas sesiones, todas realizadas de manera síncrona en la plataforma de Microsoft Teams, hacíamos un repaso de los contenidos teóricos trabajados en la asignatura, sacábamos las dudas que podían surgir y comentábamos acerca del desarrollo de los mapas. En cambio, en algunas sesiones, nos poníamos entre todos a revisar y analizar los mapas (todos los enlaces se encontraban disponibles en el Campus Virtual), generando discusiones y apuntado sus valoraciones personales. Esta práctica constante de diálogo y valoración del trabajo propio y de los demás es importante para la solidificación de un aprendizaje crítico que, además, está recogido dentro de la memoria de verificación del título como competencia básica: la elaboración y defensa de argumentos en su área de estudio (CB2).

Este cambio del parámetro de las sesiones prácticas también llevó a la alteración del porcentaje de puntos del total de la calificación dedicados al proyecto. Como todas las modificaciones que hemos implementado, la modificación de los parámetros de evaluación también fue aprobado en conjunto con los y las estudiantes. Decidimos que el proyecto debería aunar todos los puntos de la evaluación práctica de la asignatura (30\%). De la misma forma y debido a los cambios generados por la docencia no presencial y su adecuación a los sistemas de evaluación, optamos por dar más peso a la evaluación continua también en el porcentual dedicado a los contenidos teóricos y expositivos de la asignatura. Diseñamos distintas actividades

\section{MAGISTER}

Vol. 32. Núm. I: (2020). Sección extraordinaria 
para trabajar y evaluar estos contenidos y dos de ellas ${ }^{1}$ fueron incluidas en el mapa, sirviéndonos del abanico de posibilidades brindadas por las herramientas de geolocalización y el m-learning, significativamente ampliadas en aquellos momentos de forzosa no presencialidad.

Finalmente, la última sesión síncrona que tuvimos sirvió para la realización de un intercambio de impresiones sobre la actividad, sus aportaciones y carencias. Conectados simultáneamente desde sus casas. Cada alumno y alumna tuvo tiempo de abrir su mapa, explicar cómo lo había concebido y sistematizado y explicar el punto que mejor lo ilustrase (Fig. 2).

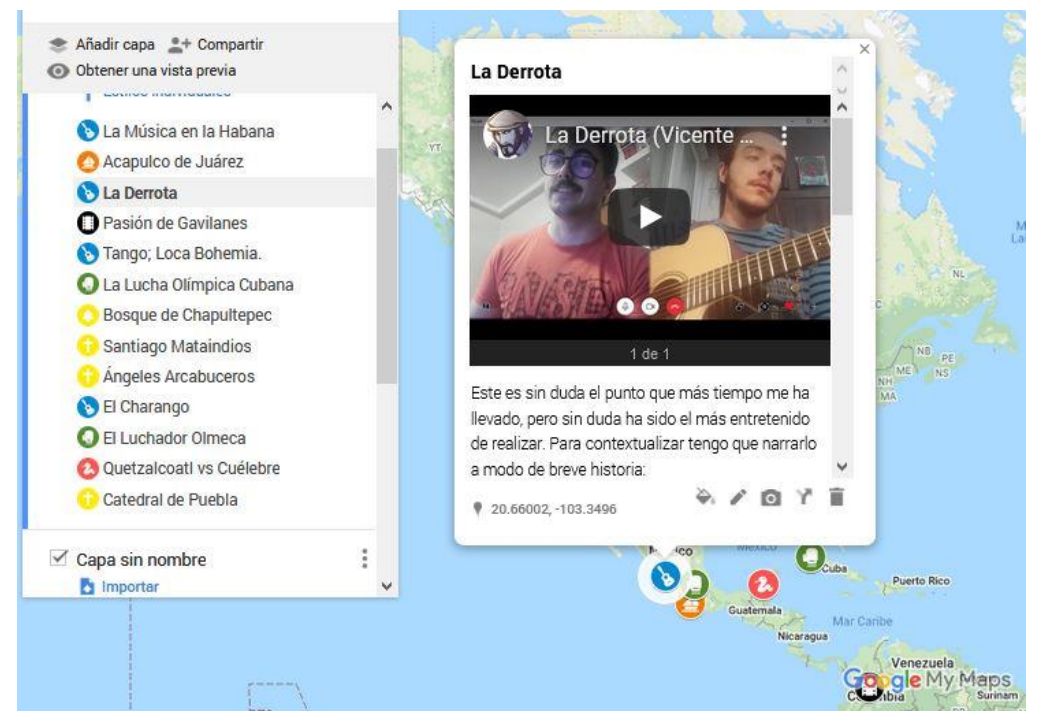

Figura 2. Ejemplo de ubicación elegida como más representativa en la presentación final del proyecto.Fuente: Captura de pantalla de My Maps

\section{Conclusiones}

La "utopía de la proximidad" que identifica Bourriaud (2006) en las obras de arte de finales del siglo XX surgió como forma de relacionarse frente a la evidente falta de conexiones del arte con la convivencia real. Los artistas, delante de un panorama social que enterraba las comunicaciones y los contactos humanos, buscaron alternativas para construir una nueva proximidad. Creemos que el gran logro del proyecto que aquí hemos descrito fue el de construir una proximidad relacional en unos tiempos tan peculiares y convulsos. Aunque el contacto fue creado mediante tecnologías y herramientas que filtran la presencialidad entendida como real, se logró construir puentes y efectuar pequeñas bifurcaciones entre alumnos y alumnas, que favorecieron no solamente el fortalecimiento de los conocimientos y su proceso de aprendizaje, pero también los lazos entre ellos y ellas. En este sentido, terminamos con una reflexión que hizo una estudiante en la evaluación final de la actividad: "he conocido más a algunas de mis compañeras leyendo sus puntos en el mapa, que durante los años que compartimos clases".

\section{Referencias}

Alejandre, S.N., Ortiz, M.J., e Izaguirre, R.C. (2018). La relación cultura geográfica - espacio cultural en la formación de la identidad cultural. Nadir: Revista electrónica de Geografía Austral,10, 1-9. Recuperado de http://revistanadir.yolasite.com/resources/ARTICULO\%20SUSEL\%20ALE JANDRE\%20NADIR.pdf (Acceso 15 de julio de 2020).

Bourriaud, N. (2006). Estética Relacional. Buenos Aires: Adriana Hidalgo Editora.

Espinosa, I.J. (2019). El giro educativo epistemológico: Pedagogía(s) Decolonial(es) y dimensión intercultural-afectiva. En Melo, A., Torres, I.J., Pons, L., y

construcción de las grandes colecciones. Los videos que produjeron se cargaron en un canal de YouTube y luego, los enlaces fueron enviados vía WhatsApp para los y las estudiante de la asignatura Conocer los pueblos prehispánicos: aspectos de la cultura y el arte, que impartimos en PUMUO (Programa para Mayores de la Universidad de Oviedo).

\section{MAGISTER}

Vol. 32. Núm. I: (2020). Sección extraordinaria 
Rivas, J.I. (coords.). Perspectivas decoloniales sobre la educación (pp. 6194). Málaga, Guarapuava: UMA Editorial, UNICENTRO.

Fombona, J. y Vázquez-Cano, E. (2017). Posibilidades de utilización de la Geolocalización y Realidad Aumentada en el ámbito educativo. Educación XXI, 20(2), 319-342.

Fundación Telefónica (2012). Guía Mobile Learning. Madrid: Fundación Telefónica. Recuperado de https://observatorio.profuturo.education/wpcontent/uploads/2016/04/Guia_MobLearning.pdf (Acceso 10 de julio de 2020).

Gros, B. y Forés, A. (2013). El uso de la geolocalización en educación secundaria para la mejora del aprendizaje situado: Análisis de dos estudios de caso. RELATEC, Revista Latinoamericana de Tecnología Educativa, 12(2), 41-53. Recuperado de https://relatec.unex.es/article/view/1193 (Acceso 12 de julio de 2020).

Leiva, J.J. y Moreno, N.M. (2015). Tecnologías de Geolocalización y Realidad Aumentada en contextos educativos: experiencias y herramientas didácticas, Revista DIM, 11(31), 1-18. Recuperado de https://www.raco.cat/index.php/DIM/article/view/291534/380014 (Acceso 10 de julio de 2020).

Perales, V. y Adam, F. (2013). Integración de GIS (sistemas de georreferenciación de la información) y localización espacial en prácticas pedagógicas y lúdicas vinculadas a museos. Arte, Individuo Y Sociedad, 25(1), 121-133. https://doi.org/10.5209/rev ARIS.2013.v25.n1.41168

Walsh, C. (2014). Lo pedagógico y lo decolonial: entretejiendo caminos. Pedagogías decoloniales. Prácticas insurgentes de resistir, (re)xistir y (re)vivir. Quito: Editorial Aby-Ayala.

*Autor de contacto: Renata Ribeiro dos Santos, ribeirorenata@uniovi.es

\section{MAGISTER}

Vol. 32. Núm. I: (2020). Sección extraordinaria 